\title{
Anthracycline-Induced Cardiac Toxicity: A Clinical Review
}

\begin{abstract}
Anthracyclines (ATCs) have a great efficacy against many types of cancer and is currently considered a cornerstone in the treatment of numerous pediatric and adult hematological and solid tumors. Great advances have been achieved after the entry of ATC group into the cancer treatment in the early 1960s, and the overall survival ratio has increased from $30 \%$ to near $70 \%$. Due to their significant role and great value in cancer therapy, which is persistent to date, ATCs are listed in the World Health Organization model list of essential medicines. The clinical use of ATC such as doxorubicin and daunorubicin can be viewed as a sort of double-edged sword. On the one hand, ATCs play an undisputed key role in the treatment of many neoplastic diseases; on the other hand, the administration of ATC is associated with the risk of severe adverse effects. The most common side effect of the ATC group is cardiotoxicity (CTX), which may limit its use and increases mortality and morbidity rates. The clinical use of ATC is limited by unique maximum total cumulative dose (approximately $350 \mathrm{mg} / \mathrm{m}^{2}$ ) limiting CTX. ATC CTX is cumulative dose-dependent and is in most of the occasions irreversible. Lowering the cumulative dose has been proved to be useful in minimize the risk of heart failure (HF), but, yet, there is a growing concern that HF might occur following doses that were thought to be safe. The average incidence of HF is around 5\% at a cumulative dose of $400 \mathrm{mg} / \mathrm{m}^{2}$ that becomes higher above $500 \mathrm{mg} / \mathrm{m}^{2}$, albeit with substantial individual variation. The newer generations ATC medications such as epirubicin, idarubicin, and mitoxantrone were thought to be safer; however, subsequent clinical studies showed more or less similar toxicity profiles. The use of cardioprotective agents (e.g., dexrazoxane and amifostine) has been associated with improved safety range; however, questions are looming on their effect on ATC antitumor effects. An overwhelming amount of clinical evidence suggests that ATCs are too good to be old. Yet, they would look much better if they caused less harm to the heart when administered as either single agents or in combination with otherwise promising new drugs. In this review article, we present a comprehensive account on the ATC and provide up to date data on their clinical use and toxicity profile. In addition, we provide a contemporary approach on the early detection, diagnosis, and treatment of ATC CTX.
\end{abstract}

Keywords: Anthracyclines, cardiac failure, daunorubicin, dexrazoxane, doxorubicin

\section{Introduction}

Anthracyclines (ATCs), a family of chemotherapeutic (CTR) drugs with efficacy against many cancers is currently considered a cornerstone in the treatment of numerous hematological and solid tumors for more than 50 years. ATCs have been discovered by Federico Arcamoni in Italy in the early 1950s. They are produced from a spore-forming, pigment-producing Streptomyces peucetius. Although they were initially produced as antimicrobial agents, they were not widely used as such, especially after the discovery of their potent anticancer properties. Great advances have been achieved after the entry of the ATC

This is an open access journal, and articles are distributed under the terms of the Creative Commons Attribution-NonCommercial-ShareAlike 4.0 License, which allows others to remix, tweak, and build upon the work non-commercially, as long as appropriate credit is given and the new creations are licensed under the identical terms.

For reprints contact: reprints@medknow.com group into the cancer treatment in the early 1960 s, and the overall survival ratio has increased from $30 \%$ to near $70 \%{ }^{[1]}$ About $60 \%$ of childhood cancer survivors are treated with ATC. Due to their major role and great value in cancer therapy, which is persistent to date, ATCs are listed in the World Health Organization model list of essential medicines. ${ }^{[2]}$ The clinical use of ATC such as doxorubicin (DOX) and daunorubicin (DNR) was associated with improved survival, but that was often accompanied by increased rate of treatment-related complications. Therefore, ATC prescription can be viewed as a sort of double-edged sword. On the one hand, ATCs play an undisputed key role in the treatment of many neoplastic diseases; on the other hand, chronic administration of ATC is associated with

\footnotetext{
How to cite this article: Abbas AA, AIAzmi AA. Anthracycline-induced cardiac toxicity: A clinical review. Indian J Med Paediatr Oncol 2019;40:465-75.
}

Adil Abdelhameed Abbas $^{1,2}$, Aeshah Abdu Mubarak AlAzmi ${ }^{2,3}$

${ }^{1}$ Department of Pediatrics, College of Medicine, King Saud Bin Abdulaziz University for Health Sciences, Riyadh, ${ }^{2}$ Princess Nourah Oncology Centre, King Abdulaziz Medical City, ${ }^{3}$ Department of Pharmacology, College of Pharmacy, King Abdulaziz University, Jeddah,

Saudi Arabia

Submitted: 29-Apr-2019 Revised: 23-Jul-2019 Accepted: 04-Aug-2019 Published: 17-Feb-2020

Address for correspondence: Dr. Adil Abdelhameed Abbas, Princess Nourah Oncology Centre, King Abdulaziz Medical City, P. O. Pox 9515, Jeddah 21423, Saudi Arabia. E-mail: adilahabbas2000@ yahoo.com.au 
severe adverse effects. The first report about ATC-related cardiotoxicity (CTX) was in 1970s where a retrospective analysis of $>4000$ patients treated with DOX containing cancer regimen was published and showed that $2.2 \%$ of the patients developed symptoms and signs of congestive heart failure (HF).$^{[3]}$ The most common side effect of ATC therapy is CTX, which may limit its use and increases mortality and morbidity rates. The clinical use of ATC is limited by unique maximum total cumulative dose (approximately $350 \mathrm{mg} / \mathrm{m}^{2}$ ) limiting CTX. ATC CTX is cumulative dose-dependent and irreversible. ${ }^{[4,5]}$ In the era of targeted therapy, the ATC, which were discovered more than half-century ago, may appear to be too old to be good. However, we are now discovering that new combination therapies often cause a higher than expected incidence of CTX, as if the newly designed drugs make the heart more vulnerable to the old one. An overwhelming amount of clinical evidence suggests that ATCs are too good to be old. Yet, they would look much better if they caused less harm to the heart when administered as either single agents or in combination with otherwise promising new drugs. ${ }^{[6]}$

\section{Epidemiology}

ATC CTX is exponentially dose-dependent. Lowering the cumulative dose of DOX has been proven to be useful in minimizing the HF risk, but yet, there is a growing concern that HF might occur after doses that were thought to be safe. The average incidence of HF is around 3.5\% at a cumulative ATC dose of $400 \mathrm{mg} / \mathrm{m}^{2}$ that becomes higher above $500 \mathrm{mg} / \mathrm{m}^{2}$, albeit with substantial individual variation. Dose-limitation strategies have reduced the incidence of ATC-related cardiac events. In a moderate cumulative DOX dose of $240-400 \mathrm{mg} / \mathrm{m}^{2}$, the incidence of asymptomatic left ventricular dysfunction (LVD) is around $25 \%$ and $35 \%$ in patients treated with a moderated cumulative dose of $240-400 \mathrm{mg} / \mathrm{m}^{2}, 7 \%-16 \%$ in patients treated with a cumulative dose of $550 \mathrm{mg} / \mathrm{m}^{2}$, and $18 \%-48 \%$ at a high cumulative ATC dose of $700 \mathrm{mg} / \mathrm{m}^{2}$.

The overall incidence of congestive HF is between $4.5 \%$ and $7 \%$. However, clinicians are facing new problems, such as asymptomatic LVD, cardiovascular events in long-term survivors, and higher than expected occurrences of CTX in patients receiving ATC together with new targeted drugs, such as the anti-ErbB2 and HER-2 antibody trastuzumab (TZB). ${ }^{[7,8]}$

Nowadays, cancer and heart diseases are the leading causes of morbidity and mortality in the industrialized world. The coexistence of cancer and cardiac disease in the same patient is more common due to aging population and improvements in the efficacy of antitumor agents. Modern treatment strategies have led to an improvement in the chances of surviving a diagnosis of cancer; however, these gains may come at a cost. Patients may experience adverse cardiovascular events related to their cancer treatment or as a result of an exacerbation of an underlying cardiovascular disease. With longer periods of survival, late effects of cancer treatment may become clinically evident years or decades after the completion of therapy. CTX is now recognized as a leading cause of long-term morbidity and mortality among cancer survivors. The current cancer therapy incorporates multiple agents whose deleterious cardiac effects may be additive or synergistic. Cardiac dysfunction may result from agents that can result in myocyte destruction, such as with ATC use, or from agents that appear to transiently affect the left ventricular contractility. In addition, cancer treatment may be associated with other cardiac events, such as severe treatment-induced hypertension and vasospastic and thromboembolic ischemia, as well as rhythm disturbances, including QTc prolongation that may be rarely life-threatening. Early and late effects of chest radiotherapy (XRT) can lead to XRT-induced heart disease, including pericardial disease, myocardial fibrosis, cardiomyopathy, coronary artery disease (CAD), valvular disease, and arrhythmias, in the setting of myocardial fibrosis. ${ }^{[9]}$

Cardio-oncology is a new discipline that focuses on screening, monitoring, and treating cardiovascular disease during and after cancer treatment. The discipline of cardio-oncology has developed in response to the combined decision-making necessary to optimize the care of cancer patients, whether they are receiving active treatment or are long-term survivors. A baseline cardiovascular risk assessment is essential. For high-risk patients, a tailored and detailed plan for cardiovascular management throughout treatment and beyond should also be established. ATC and/or TZB-containing CTR and chest-directed XRT are well-known cardiotoxic cancer therapies. Monitoring for the development of subclinical CTX is crucial for the prevention of clinical HF. ${ }^{[10]}$

The Canadian Cardiovascular Society authored by a pan-Canadian expert group of health-care providers and commissioned by the Canadian Cardiovascular Society produced guidelines for evaluation and management of cardiovascular complications of cancer therapy. These guidelines are intended to guide the care of cancer patients with established cardiovascular disease or those at risk of experiencing toxicities related to cancer treatment. It provides guidance to clinicians on contemporary best practices for the cardiovascular diseases. The guidelines include recommendations and important management considerations with a focus on four main areas: identification of the high-risk population for CTX, detection, and prevention of CTX; treatment of CTX; and a multidisciplinary approach to cardio-oncology. All recommendations align with the Grading of Recommendations Assessment, Development, and Evaluation system. Key recommendations for which the panel provides a strong level of evidence include: (1) that routine evaluation of traditional cardiovascular risk factors and optimal treatment of preexisting cardiovascular disease 
be performed in all patients before, during, and after receiving cancer therapy; (2) that initiation, maintenance, and/or augmentation of antihypertensive therapy be instituted per the Canadian Hypertension Educational Program guidelines for patients with preexisting hypertension or for those who experience hypertension related to cancer therapy; and (3) that investigation and management follow the current Canadian Cardiovascular Society HF guidelines for cancer patients who develop clinical HF or an asymptomatic decline in the left ventricular ejection fraction (LVEF) during or after cancer treatment. ${ }^{[11]}$

\section{Types of Anthracycline Products and Their Mechanism of Action}

ATC rank among the most effective anticancer drugs ever developed. The first ATCs were isolated from the pigment-producing $S$. peucetius early in the 1960s and were named DOX) and DNR. DOX and DNR share aglyconic and sugar moieties called daunosamine. The only difference between DOX and DNR is that the side chain of DOX terminates with a primary alcohol, whereas that of DNR terminates with a methyl. This minor difference has important consequences on their spectrum of activity. Whereas DOX is an essential component in the treatment of breast cancer, childhood solid tumors, soft-tissue sarcomas, and aggressive lymphomas, DNR shows activity in acute lymphoblastic or myeloblastic leukemias. As with any other anticancer agent, the clinical use of both DOX and DNR soon proved to be hampered by serious problems such as the development of resistance in tumor cells or toxicity in healthy tissues.

When administered intravenously, ATCs are quickly distributed to the various tissues and reach the highest concentration in the lungs, liver, bone marrow, and spleen. Except for idarubicin (IDA), ATC products do not cross the blood-brain barrier. The average half-life is $>24 \mathrm{~h}$. Metabolism is mainly hepatic, where $60 \%-80 \%$ is excreted into the bile and $20 \%-40 \%$ excreted in the urine.

Despite extensive clinical utilization, the mechanisms of action of ATCs in cancer cells remain controversial. In a seminal commentary, the following mechanisms were considered: (1) intercalation into deoxyribonucleic acid (DNA), leading to inhibited synthesis of macromolecules; (2) generation of $\mathrm{O}_{2}$ free radicals, leading to DNA damage or lipid peroxidation; (3) DNA binding and alkylation; (4) DNA cross-linking; (5) interference with DNA unwinding or DNA strand separation and helicase activity; (6) direct membrane effects; (7) initiation of DNA damage through inhibition of topoisomerase II; and (8) induction of apoptosis in response to topoisomerase II.

ATCs are topoisomerase enzyme inhibitors. Recent data suggest that they intercalate between the base pairs of the DNA or ribonucleic acid (RNA) and inhibits DNA replication and RNA transcription resulting in decrease replication of cancer cells. ${ }^{[4,12]}$

The last 2 decades have witnessed numerous attempts to identify a novel ATC that proves superior to DOX and DNR in terms of activity and/or cardiac tolerability. The search for the "better ATC" has resulted in some 2000 analogs. Yet only a few analogs have reached the stage of clinical development and approval; among them, epirubicin (EPI) and IDA enjoy popularity as useful alternatives to DOX or DNR, respectively. EPI is a semisynthetic derivative of DOX obtained by an axial-to-equatorial epimerization of the hydroxyl group at $\mathrm{C}-4$ in daunosamine. It is because of these metabolic changes that EPI was soon used at cumulative doses almost double those of DOX, resulting in equal activity but not in increased CTX. In practice, early studies of patients with advanced breast cancer demonstrated that the median doses to the development of laboratory indices of CTX were $935 \mathrm{mg} / \mathrm{m}^{2}$ of EPI compared with $468 \mathrm{mg} / \mathrm{m}^{2} \mathrm{DOX}$; however, the rate of development of cardiomyopathy remained the same. IDA, an analog obtained from DNR after removal of the 4-methoxy group in ring $\mathrm{D}$, is active in acute myelogenous leukemia (AML), multiple myeloma (MM), non-Hodgkin's lymphoma, and breast cancer. The broader spectrum of activity of IDA compared with DNR may be attributed to increased lipophilicity and cellular uptake and improved stabilization of a ternary drug-topoisomerase. In addition, IDA may be administered orally (with $10 \%-30 \%$ bioavailability, and in vitro studies have indicated that it might be more effective than DNR in tumor cell lines displaying the multidrug resistance phenotype. There is some controversy about whether IDA offers advantages over DOX or DNR in regard to cardiac toxicity. Some authors concluded that oral IDA does not induce CTX, not even in patients previously exposed to DOX or EPI. In contrast, others have shown that IDA decreases LVEF in ATC-nai"ve patients and causes Congestive $\mathrm{HF}$ in patients with preexisting cardiovascular disease or previous ATC treatment. Only a few more ATCs have attained clinical approval; including pirarubicin, aclacinomycin A (aclarubicin), and mitoxantrone (MXT) (a substituted aglyconic anthraquinone). Both pirarubicin and aclarubicin demonstrate only modest improvements over DOX and DNR in terms of drug resistance. Pirarubicin, a 4-tetrahydropyranyl has been reported to induce much less CTX than DOX in animal models, but studies in women with metastatic breast cancer have indicated that it may cause a significant decrease in LVEF or full-blown congestive $\mathrm{HF}$ at cumulative doses of $460 \mathrm{mg} / \mathrm{m}^{2}$ or $500 \mathrm{mg} / \mathrm{m}^{2}$, respectively. Aclarubicin, a trisaccharide ATC, was shown to be active and cardiac-tolerable in adult patients with AML. However, aclarubicin induced late cardiac events in a Phase II study in adult patients with refractory AML or acute lymphoblastic leukemia and proved to be inactive in women with metastatic breast cancer. MXT is active in breast cancer, acute promyelocytic 
leukemia, AML, and androgen-independent prostate cancer. Early reports indicated that MXT was less cardiotoxic than other ATCs, but this conclusion has been challenged in more recent studies. Moreover, MXT causes chronic CTX in patients with worsening relapsing-remitting or secondary progressive multiple sclerosis. ${ }^{[13-15]}$

Alongside CTX, ATCs are associated with other forms of systemic side effects such as bone marrow suppression with resultant anemia, neutropenia and thrombocytopenia, severe mucositis, necrotizing colitis, and extravasation necrosis.

The new liposomal-encapsulated DOX has been shown to be at least as active as free DOX in experimental models, and Phase I and II human studies indicate that this novel strategy of drug delivery may have less myocardial toxicity. Few clinical trials in adult science technology studies have been published until now, with disappointing and often contrasting results. Liposomal DOX has not been tested at doses adequate to exploit the antitumor effects of the drug, being the reached dose-intensity being even lower than those deemed critical for obtaining optimal responses to free DOX.

The newer pegylated liposomal doxorubicin (PLD) is the first drug produced by nano technology to be approved for cancer therapy. PLD is a complex formulation of DOX based on pharmaceutical nanotechnology with unique pharmacokinetic and pharmacodynamic properties. Its long circulation time with stable retention of the payload and its accumulation in tumors with high vascular permeability both result in important advantages over conventional CTR. The ability of PLD to buffer several undesirable side effects of DOX, including a major risk reduction in cardiac toxicity, is now well-established and confers a major added value in a number of disease conditions. PLD is approved for the treatment of ovarian cancer, breast cancer, MM, and Kaposi sarcoma. In addition, clinically significant antitumor activity of PLD has been reported in a number of other cancer types, including lymphomas and soft-tissue sarcomas. In spite of this, PLD has not replaced conventional DOX in common applications such as the adjuvant and neoadjuvant treatment of breast cancer, and its use in the clinic has not become as widespread as one may have predicted. ${ }^{[16,17]}$

These remarks on the activity and toxicity of most commonly used ATC compounds are meant to indicate that a better ATC has yet to come. It is therefore not surprising that relatively old drugs such as DOX and DNR remain the focus of clinical and preclinical research aimed at improving our appraisal of their mechanisms of activity or toxicity and at identifying new strategies for better use in cancer patients. Likewise, the search for new analogs or formulations continues unabated. ${ }^{[18-21]}$

\section{Mechanism of Cardiac Injury}

ATCs are among the most common CTR agents that have been recognized to cause CTX. Although various forms of cardiac damage have been demonstrated with the use of these drugs in experimental studies, it is not yet clear how these translate to the clinical setting. Despite more than five decades of investigation, the exact pathogenic mechanisms responsible for ATC CTX have not yet been completely elucidated. In the last few years, several experimental and clinical investigations provided new information and perspectives on ATC-related CTX. ATC induces progressive myocardial cell death with continuous administration, and that may continue for years after stopping treatment. In particular, molecular mechanisms of CTX have been better elucidated. Although there may be many causes of ATC-induced CTX, a large body of evidence points to $\mathrm{O}_{2}$ free-radical-mediated myocyte damage. One-electron redox cycling of the quinone moiety has long been known to form reactive $\mathrm{O}_{2}$ species (ROS) in excess of the limited antioxidant defenses of cardiomyocytes; therefore, ATC CTX was perceived as a one-way process in which redox cycling of the quinone always primed cardiomyocytes to oxidant stress and death. An alternative process is the process in which peroxidases and quinone-derived hydrogen peroxide oxidize the hydroquinone moiety of ATC. Such a process was initially thought to amplify the CTX induced by ATC. Oxyferrous myoglobin could be subsequently identified as the primary catalyst of ATC oxidation in cardiomyocytes and be shown to induce an ATC chemical degradation that diminished the cellular levels and toxicity of active parent compounds. Many aspects of ATC degradation remain obscure or only partially understood; nevertheless, it is not too naive to conclude that ATCs are degraded and inactivated as a result of ROS production from their own redox cycling. ATC redox reactions might therefore be viewed as two-way processes in which oxidative stress mediates both the death and survival of cardiomyocytes. ${ }^{[22]}$

Appropriate characterization of potential candidates for ATC-based therapies is essential to decide whether to administer these drugs. Hopefully, new information from genetic profiling will help to identify patients who are at a high risk of developing CTX. ${ }^{[23]}$ DOX-induced CTX is probably mediated through the formation of DOX - iron - complex. Lipshultz et al. reported increased frequency of ATC myocardial injury in carriers of hemochromatosis gene C282Y. Screening of newly diagnosed cancer patients may identify those at risk of developing CTX. ${ }^{[24]}$

\section{Clinical Forms of Anthracycline Cardiotoxicity}

Up to $50 \%$ of patients treated with ATC containing regimens will demonstrate some form of CTX. ATC-induced CTX can manifest as almost any cardiovascular disease. LVD is the most typical manifestation of ATC-induced CTX and can lead to congestive HF. Nearly 25\% of patients treated with ATC develop asymptomatic LVD, and almost half of them progress over months to years 
to develop congestive HF. Few patients however exhibit electrocardiogram (ECG) alterations including prolongation of QT interval, development of varies atrial and ventricular arrhythmias, and increasing the chances of sudden death. The effects of the interaction of ATC with the monoclonal antibody against HER2/neu (Erb-2) tTZB could potentiate the cardiotoxic effects. The mechanism seems to be through the affection of ventricular repolarization through a set of different mechanisms. Interference on human gene potassium ion channels (HERG $\mathrm{K}+$ ) seems to be a common mechanism for many of these drugs. ${ }^{[25]}$

LVD may be permanent, due to the formation of myocardial scar, or it may be reversible after revascularization. Reversible LV dysfunction occurs when the myocardium is viable but dysfunctional (reduced contractility). Since only patients with dysfunctional but viable myocardium benefit from revascularization, the identification and quantification of the extent of myocardial viability are an important part of the work-up of patients with cardiac dysfunction. ${ }^{[26]}$

Higher mortality rates are reported because of CAD in cancer survivors. Cardiotoxic CTR agents and/or mediastinal XRT are additional risk factors for the development of CAD. Mediastinal XRT and ATC containing CTR regimens are commonly used to treat patients with lymphoma. ATC used in cancer treatment and XRT may cause deleterious effects on contractile capacity and conduction system of the heart. Approximately 10 years after the completion of all therapies, the cardiovascular disease risk peaks in patients who survived cancer and CAD becomes a problem. Coronary computed tomography angiography (CCTA) is a useful diagnostic tool in determining early CAD. ${ }^{[27]}$ A St. Jude Children's Research Hospital report of 31 survivors of Hodgkin's lymphoma was published Mulrooney et al. in 2014. Thirteen of those patients $(42 \%)$ were treated with XRT only, and 18 of whom (58\%) were treated with multimodal therapy, underwent CCTA, echocardiography, ECG, and treadmill stress testing. The obstructive CAD was defined as $\geq 50 \%$ occlusion of the left main or $\geq 70 \%$ occlusion of the left anterior descending, left circumflex, or right coronary arteries on CCTA. Echocardiograms with resting wall motion abnormalities or an ejection fraction (EF) $<50 \%$; ECGs with Q waves, ST abnormalities without Q waves, or T-wave abnormalities without $\mathrm{Q}$ waves; and a J-point depression of $\geq 1 \mathrm{~mm}$ with a horizontal or down-sloping ST-segment on stress testing were considered abnormal.

The prevalence of obstructive CAD in participants (median age, 40 years [range, 26-55 years]; median time from cancer diagnosis, 24 years [range, 17-39 years]) was 39\%, with 39 plaques detected among 12 survivors. Three participants $(10 \%)$ treated with XRT only had 4 obstructive lesions; 9 patients $(29 \%$; 5 of whom were treated with XRT only and 4 of whom were treated with multimodal therapy) had nonobstructive lesions.
Approximately $15 \%$ of lesions involved the left main, $21 \%$ involved the proximal left anterior descending, $18 \%$ involved the proximal right coronary, and $13 \%$ involved the proximal left circumflex arteries. Of the 12 participants found to have CAD by CCTA, 7 had a positive ECG, 1 had a positive echocardiogram, and 1 had a positive stress test. The study concluded that CCTA identified CAD in a substantial percentage of survivors of Hodgkin's lymphoma and may be an effective screening modality for such patients. ${ }^{[28]}$

Congestive $\mathrm{HF}$ is a complex clinical syndrome that can result from any functional or structural cardiac disorder that impairs the ventricle's ability to fill or eject blood. Since there is no definitive diagnostic test for HF, it remains a clinical diagnosis that is largely based on a careful history and physical examination and supported by ancillary tests such as chest radiograph, electrocardiogram (ECG), and echocardiography. $\mathrm{HF}$ is a common disease, affecting approximately 5 million people in the United States, and it occurs predominately in the elderly, with almost $80 \%$ of cases occurring in patients over the age of 65 years. The development of congestive $\mathrm{HF}$ in children is uncommon and indicates the presence of a serious underlying problem. Several studies have found that congestive $\mathrm{HF}$ is associated with a 2-year mortality rate of approximately $45 \%-50 \%$, which approaches that of many malignancies. ${ }^{[29]}$ There are two mechanisms of reduced cardiac output and HF: systolic dysfunction and diastolic dysfunction. The most common causes of systolic dysfunction (defined by an LVEF of $<50 \%$ ) are ischemic heart disease, idiopathic dilated cardiomyopathy, hypertension, and valvular heart disease. Diastolic dysfunction (defined as dysfunction of LV filling with preserved systolic function) may occur in up to $40 \%-50 \%$ of patients with HF, it is more prevalent in women, and it increases in frequency with each decade of life. Diastolic dysfunction can occur in many of the same conditions that lead to systolic dysfunction. The most common causes are hypertension, ischemic heart disease, hypertrophic cardiomyopathy, and restrictive cardiomyopathy. ${ }^{[26,30]}$

\section{Clinical Presentation}

Up to $50 \%$ of ATC-treated patients will demonstrate some form of CTX. ATC-induced CTX can manifest as almost any cardiovascular disease. The most common are congestive HF and asymptomatic LVD. Patients are at an increased risk of sudden death due to severe cardiac arrhythmias because of the high prevalence of predisposing risk factors such as electrolytic abnormalities, starvation, and concomitant medications. ATC induces progressive CTX and cardiac dysfunction with a progressive reduction in the LV EF. Reduction in the LVEF may result in cardiac decompensation and development of congestive HF. Stabilization of the LVEF may occur with discontinuation of ATC therapy with little room for improvement. 
After exposure to ATC medications, the incidence of cardiac LV function abnormalities increases with time. Higher cumulative ATC dose is the most clinical factor that predicts the development of HF. Once diagnosed with $\mathrm{HF}$ the 5-year survival drops to $<50 \%$, and the mortality rate is as high as $20 \%$. ATC-induced congestive HF is usually due to permanent changes in the myocardium. The changes are most consistent with the contractile failure cardiomyopathy. ${ }^{[3,31]}$

\section{Risk Factors}

ATCs are used in all age ranges, thereby exposing a broad population of patients to the development of CTX. For some treated patients, ATCs affect cardiac muscle, resulting in cardiomyopathy. The type and degree of cardiomyopathy, as well as when, during, or after treatment, the condition occurs, are dependent on what risk factors are present. Age is a major risk factor. Children and adults may develop restrictive and dilated cardiomyopathy. The length of subsequent survival and amount of subsequent somatic growth may influence late ATC-associated cardiac outcome. The risk of developing HF is modified by the presence of certain risk factors that reduce cardiac tolerance to ATC. Age and female gender seem to have an important role in the ATC-induced CTX. ATC-induced CTX can be divided, on the basis of when it started, into acute, subacute and progressive late, chronic form. Early CTX, occurring during or within 1 year of completion of treatment, is the largest risk factor for the development of late CTX, which occurs beyond a year of completion of treatment. Risk factors, which appear to be specific for early CTX in children, include black race, trisomy 21 , and the use of amsacrine after ATC therapy. More cardiotoxic effects are seen in survivors of childhood cancer; the longer from completion of treatment a patient is followed. Cumulative and peak ATC doses affect adults and children alike, and CTX occurs early and late. In adults, the left ventricular contractility is affected by ATC. Children may manifest as impairment of the left ventricular contractility and increased afterload due to thinning of left ventricular walls. Patients with an early presentation of depressed left ventricular contractility are likely to show the progression of cardiac disease with time. In addition, female gender appears to affect early and late CTX in both adults and children, although this risk factor has been described predominantly in the survivors of childhood cancer. ${ }^{[32,33]}$

\section{Diagnosis}

LFD is the most typical manifestation of ATC-induced CTX and can lead to HF. Detecting a decreased LVEF after cancer therapy might be a late finding; therefore, earlier markers of cardiac injury are being actively explored. Abnormal myocardial strain and increased serum cardiac biomarkers (e.g., troponins and natriuretic peptides) are possible candidates for this purpose. Early diagnosis has also improved through the use of advanced noninvasive cardiac imaging techniques, and emerging data indicate a genetic predisposition to develop ATC-related CTX. ${ }^{[10,23,34]}$ Standard M-mode echocardiography and tissue Doppler imaging (TDI) are generally a very sensitive tests in detecting ventricular dysfunction. Echocardiograms were routinely used to monitor cardiac function after ATC treatment. Nevertheless, indices chosen to assess cardiac toxicity vary significantly among different centers, and no uniform protocol has been accepted as ideal. Common echocardiographic measures include LVEF; peak systolic myocardial longitudinal, radial, and circumferential strain and echocardiographic markers of diastolic function. LVEF measurement by echocardiography and multigated radionuclide angiography is the most common diagnostic approach to detect cardiac damage, but it identifies a late manifestation of myocardial injury.

Common abnormal echocardiographic findings include diastolic dilation of the left ventricle, thinner interventricular septum, decreased left ventricular mass in females, follow-up dependent dilation of the LV in systole, and follow-up dependent decrease in EF. TDI abnormalities included signs of early diastolic dysfunction, and myocardial hypertrophy were also found in structures that appeared normal by M-mode echocardiography. ${ }^{[35]}$

A retrospective study included children with Ewing sarcoma treated according to the standard protocol containing significant cumulative doses of anthracycline compounds at the Children's Cancer Hospital Egypt was reported by Moussa et al. All echocardiograms and related clinical assessments were reviewed. The study included 149 patients (median age 11 years; range 1-18 years). Although all patients had a reduced EF compared with their baseline echocardiogram, only 39 patients developed CTX (26\%): 43\% acute-onset, 36\% chronic early-onset, and $21 \%$ chronic late-onset. There was no statistically significant association between the frequency of myocardial dysfunction and risk factors, including age, sex, follow-up duration, cumulative DOX dose, and mediastinal XRT. Over one-third $(39 \%)$ of the patients with cardiac toxicity regained normal cardiac parameters, whereas seven patients died of acute cardiac toxicity. In this group of patients, echocardiography was a useful screening test for ATC-induced cardiac toxicity before and during CTR identified myocardial dysfunction. ${ }^{[32]}$

Early noninvasive imaging techniques are needed for the diagnosis and monitoring of cardiotoxic effects. Although echocardiography and cardiac magnetic resonance imaging (MRI) are the most commonly used imaging techniques for CTX assessment, greater attention is focused on new nuclear cardiologic techniques, which can identify high-risk patients in the early stage and visualize the pathophysiologic process at the tissue level before clinical manifestation. ${ }^{[36]}$ 
In October 2010, the Medical Advisory Secretariat (MAS) of Canada published a report as to the most useful noninvasive techniques to detect myocardial viability assessment. Five key noninvasive cardiac imaging technologies were enumerated: positron emission tomography (PET), cardiac MRI, dobutamine echocardiography, dobutamine echocardiography with contrast, and single-photon emission computed tomography (SPECT) scan. An earlier 2005 review conducted by MAS determined that PET scan was more sensitive than dobutamine echocardiography and SPECT and dominated the other imaging modalities from a cost-effective standpoint. However, there was inadequate evidence to compare PET and cardiac MRI imaging. LVD may be permanent, due to the formation of myocardial scar, or it may be reversible after revascularization. Reversible LVD occurs when the myocardium is viable but dysfunctional (reduced contractility). Since only patients with dysfunctional but viable myocardium benefit from revascularization, the identification and quantification of the extent of myocardial viability is an important part of the work-up of patients with HF when determining the most appropriate treatment path. Various noninvasive cardiac imaging modalities can be used to assess patients in whom the determination of viability is an important clinical issue, specifically: dobutamine echocardiography, stress echocardiography with contrast, SPECT scan using either technetium or thallium, cardiac MRI scan, and PET scan. ${ }^{[37]}$ Stress echocardiography can be used to detect viable myocardium. During the infusion of low-dose dobutamine $(5-10 \mu \mathrm{g} / \mathrm{kg} / \mathrm{min})$, an improvement of contractility in hypokinetic and akinetic segments is indicative of the presence of viable myocardium. Alternatively, a low-high dose dobutamine protocol can be used in which a biphasic response characterized by improved contractile function during the low-dose infusion followed by a deterioration in contractility due to stress-induced ischemia during the high dose dobutamine infusion (dobutamine dose up to $40 \mathrm{ug} / \mathrm{kg} / \mathrm{min}$ ) represents viable tissue. Newer techniques, including echocardiography using contrast agents, harmonic imaging, and power Doppler imaging may help to improve the diagnostic accuracy of echocardiographic assessment of myocardial viability. Intravenous contrast agents, which are high molecular weight inert gas microbubbles that act like red blood cells in the vascular space, can be used during echocardiography to assess myocardial viability. These agents allow for the assessment of myocardial blood flow (perfusion) and contractile function (as described above), as well as the simultaneous assessment of perfusion to make it possible to distinguish between stunned and hibernating myocardium. SPECT scan can be performed using thallium-201 (Tl-201), a potassium analog, or technetium-99 m labeled tracers When Tl-201 is injected intravenously into a patient, it is taken up by the myocardial cells through regional perfusion, and Tl-201 is retained in the cell due to sodium/potassium ATPase pumps in the myocyte membrane. Viable tissue is identified if the delayed images exhibit significant fill-in of defects identified in the initial scans ( $>10 \%$ increase in uptake) or if defects are fixed, but the tracer activity is greater than $50 \%$. PET scan is useful in detecting myocardial viability. However, the only licensed radionuclide used in PET imaging for viability assessment is F-18 fluorodeoxyglucose (FDG). During FDG-PET scan, the radionuclides are injected into the body, and as they decay, they emit positively charged particles (positrons) that travel several millimeters into tissue and collide with orbiting electrons. This collision results in annihilation where the combined mass of the positron and electron is converted into energy in the form of two $511 \mathrm{keV}$ gamma rays, which are then emitted in opposite directions $\left(180^{\circ}\right)$ and captured by an external array of detector elements in the PET gantry. Computer software is then used to convert the radiation emission into images. Cardiac MRI is a noninvasive, X-ray free technique that uses a powerful magnetic field, radiofrequency pulses, and a computer to produce detailed images of the structure and function of the heart. ${ }^{[37]}$

Nuclear cardiologic techniques that visualize pathophysiologic processes at the tissue level could detect myocardial injury at an earlier stage. These techniques may give the opportunity for timely intervention and provide insights into the mechanisms and pathophysiology of CTX caused by anticancer agents. Both first-order functional imaging techniques (visualizing mechanical [pump] function), such as $(99 \mathrm{~m})$ Tc multigated radionuclide angiography and $(99 \mathrm{~m}) \mathrm{Tc}$ gated blood-pool SPECT, and third-order functional imaging techniques (visualizing pathophysiologic and neurophysiologic processes at the tissue level) are discussed. Third-order functional imaging techniques comprise (123) I-metaiodobenzylguanidine scintigraphy, which images the efferent sympathetic nervous innervations; sympathetic neuronal PET, with its wide range of tracers; (111) In-antimyosin, which is a specific marker for myocardial cell injury and necrosis; (99 m) Tc-annexin V scintigraphy, which visualizes apoptosis and cell death; fatty-acid-use scintigraphy, which visualizes the storage of free fatty acids in the lipid pool of the cytosol (which can be impaired by cardiotoxic agents); and (111) In-TZB imaging, to study TZB targeting to the myocardium. ${ }^{[38]}$

As individuals exposed to cardiotoxic ATC therapy are at high risk of developing $\mathrm{HF}$, an important method for preventing $\mathrm{HF}$ is the avoidance or minimization anthracycline exposure and avoiding the use of concomitant cardiotoxic therapies. Decisions must balance the antitumor efficacy of the treatment with its potential CTX. If patients develop cardiac dysfunction or HF, they should then be treated in accordance with established guidelines of treatment of HF. ${ }^{[10]}$

\section{Monitoring}

The management of cardiovascular risk factors and periodic screening with echocardiography, cardiac 
imaging, and biomarkers should be considered in high-risk cancer survivors, especially those who received ATC therapy. ${ }^{[10]}$ LVEF measurement by technetium-99 multigated radionuclide angiography is regarded as the gold standard to measure CTX in adult patients. It identifies LVD with high reproducibility and low interobserver variability. A decrease in LVEF, however, is a relatively late manifestation of myocardial damage.

Attempts to ameliorate ATC CTX have been directed toward (1) decreasing myocardial concentrations of ATC and their metabolites, (2) developing less cardiotoxic analogous, and (3) concurrently administering cardioprotectants to attenuate the effects of ATC on the heart. Much progress has been made in terms of monitoring of clinical and subclinical ATC CTX, finding alternative schedules, introducing special carriers of ATC, and using cardioprotective agents. With all these effects and with results of ongoing and future trials, we hope to be able to reduce further or even eliminate ATC-induced CTX. ${ }^{[39,40]}$

\section{Management}

In general, there are three options for the treatment of ATC-induced HF: medical treatment, heart transplantation (HT), and revascularization for those with $\mathrm{CAD}$ as the underlying cause. Concerning medical treatment, despite recent advances, mortality remains high among treated patients, while HT is affected by the limited availability of donor hearts and consequently has long waiting lists. The third option, revascularization, is used to restore the flow of blood to the heart through coronary artery bypass grafting or, in some cases, through minimally invasive percutaneous coronary interventions (balloon angioplasty and stenting). Both methods, however, are associated with important perioperative risks, including mortality, so it is essential to properly select patients for this procedure. ${ }^{[37]}$

Enalapril has been used for the treatment in childhood cancer survivors with asymptomatic LVD. Although there is some evidence that enalapril temporarily improves one parameter of cardiac function (left ventricle end-systolic wall stress), it is unclear whether it improves clinical outcomes. Enalapril was associated with a higher risk of dizziness or hypotension and fatigue. Clinicians should weigh the possible benefits with the known side effects. ${ }^{[41]}$

The protective effect of carvedilol was examined in clinical trials against placebo in ATC-treated adult patients with hematological malignancies and proved effect in improving systolic and diastolic cardiac function. ${ }^{[42]}$ Numerous molecules were investigated to protect against ATC-induced CTX. In a murine study conducted by Ibrahim et al., the authors reported that telmisartan and captopril has shown biochemical cardioprotective efficacy against ATC-induced CTX. ${ }^{[43]}$ Investigators have also focused on the effect of beta-blockage and renin-angiotensin aldosterone system inhibitors and successfully demonstrated their positive effects on ATC CTX. The main mechanisms in this protective effect are the anti-fibrotic and antioxidative effects of these agents. On the other hand, use of agents such as digitoxin, ouabain, strophanthin, dexrazoxane, and Vitamin $\mathrm{E}$ has been examined in several experimental studies. $^{[44]}$

\section{Effects of Cardioprotective Agents}

Many therapeutic modifications are being tried to reduce the risk of ATC-induced cardiomyopathy. The newer compounds IDA and MZN have been used instead of the older DNR and DOX, but evidence does not justify their use as neither compound was proven to eliminate or reduce the risk. The newer generation liposomal encapsulated DOX (LED) is somewhat promising. This product has longer circulation time in the body because it is cleared less by phagocytes. LED has a longer have life, smaller volume of distribution, slower plasma clearance, and a higher concentration at the cancer site. The drug concentration reached lower level at the heart. Therefore, it is presumed to have less cardiac effect, and therefore, patients may tolerate higher cumulative doses, however, this was not confirmed in randomized trials. ${ }^{[41,45]}$

The cardioprotective agent dexrazoxane (DRX) was discovered by Kurt Hellman in Oxford in 1972. DRX is a weak topoisomerase inhibitor. It is a derivative of EDTA, a potent intracellular iron chelator. DRX is converted intracellularly into a ring-opened chelating agent that interferes with iron-mediated $\mathrm{O}_{2}$ free radical generation, decrease iron metal complex formation, and hence reduce ATC-induced myocyte death. DRX is indicated for use in patients receiving ATC cumulative dose equal or in excess to $300 \mathrm{mg} / \mathrm{m}^{2}$. For maximum protective effect, DRX should be administered in a 10: 1 ratio and just before the ATC administration. This has been several questions as to the efficacy and safety of DRX in children the led to suspend its use in the pediatric population. There were high concerns about increased risk of infection, prolong myelosuppression, and the development of second malignant neoplasms. Lipshultz et al., however, have shown that the use DRX reduces the risk of congestive $\mathrm{HF}$ in children treated with ATC. The study has also proved that concurrent use of etoposide with DRX is associated with increased risk of secondary AML and the use of concurrent cranial XRT may increase the risk for subsequent development of brain tumors. ${ }^{[46]}$ A Children's Oncology Group study published in 2016 have shown that after extended follow-up, the use of DRX was not associated with increased rate of late mortality in long-term survivors of ALL, AML, and lymphoma. ${ }^{[47]}$

In recent years, investigators focused on the protective effects of melatonin, which has a potent antioxidant action in the side effects of ATC. Studies conducted by Bilginoğlu et al., successfully demonstrated that melatonin has positive 
effects on the protection of the heart against ATC-induced CTX in rats. ${ }^{[44]}$

Different infusion rates of DOX have been used for treating human malignancies. Organ toxicity after DOX infusion is a major issue in treatment disruption. However, whether different DOX infusion rates induce different toxicity is still unknown. In this study, the toxicity effects of different DOX infusion rates were examined in the early phase of organ toxicity. Thirty-six rats were randomly divided into 5-, 15-, and 30-min infusion rate groups. A single dose of DOX $(8.3 \mathrm{mg} / \mathrm{kg})$ was administered at different intravenous infusion rates. Blood samples were collected from the femoral artery at 1, 3, 6, 9, 12, 18, 24, 36, and $48 \mathrm{~h}$ after DOX administration. The blood cell count and blood biochemistry were analyzed. The liver, kidney, and heart were removed for pathological examinations after the rats were sacrificed. Our findings show that the 30-min group had higher injury markers in the liver (glutamic oxaloacetic transaminase and glutamic pyruvic transaminase), kidneys (blood urea nitrogen and creatinine), and heart (creatine phosphokinase-MB and lactate dehydrogenase) and had higher tumor necrosis factor-alpha and interleukin six levels than did the other groups. The 30-min group also had more severe damage according to the pathological examinations. In conclusion, slower infusion of DOX induced a higher inflammatory response and greater organ damage. ${ }^{[48]}$ A randomized controlled trial reported by van Dalen et al. showed that an ATC infusion duration of $6 \mathrm{~h}$ or longer reduces the risk of clinical HF, and it seems to reduce the risk of subclinical cardiac damage in adult cancer patient. No significant difference was identified in the occurrence of clinical HF in participants treated with a DOX peak dose of $<60 \mathrm{mg} / \mathrm{m}^{2}$ or $60 \mathrm{mg} / \mathrm{m}^{2}$ or more. ${ }^{[49]}$ Cardiac performance was assessed by echocardiography in children who were relapse-free survivors of the treatment for ALL. They received the same cumulative ATC dose (daunorubicin $180 \mathrm{mg} / \mathrm{m}^{2}$ ) either by bolus injection (UKALL $\mathrm{X}$ protocol, $n=40$ ) or by infusion (UKALL XI protocol, $n=71$ ) with a follow-up of $5.3 \pm 2.0$ and $5.4 \pm 1.0$ years, respectively. On analysis, both the bolus administration and infusion groups showed similar mild impairment of the cardiac performance, characterized by increased left ventricular end-systolic stress and impaired LV function. In conclusion, subclinical abnormality of the left ventricular performance was confirmed in both groups despite the relatively modest cumulative ATC dose received. The study was unable to demonstrate an advantage of ATC administration by 6-h infusion with respect to late CTX at this dose. ${ }^{[50]}$

\section{Prognosis}

The most feared complication of ATC therapy is CTX. Echocardiograms were routinely used to monitor cardiac function after ATC treatment. Nevertheless, indices chosen to assess cardiac toxicity vary significantly among different centers. Children survivors of cancer previously treated with ATC compounds have 5-15-fold increased risk for developing congestive $\mathrm{HF}$. A retrospective study included children with Ewing sarcoma treated according to standard protocol (POG \#9354/CCG \#7942, Regimen A) containing significant cumulative doses of ATC compounds $\left(375 \mathrm{mg} / \mathrm{m}^{2}\right)$ at Children's Cancer Hospital Egypt over a period of 4 years. The cardiac assessment was done using echocardiography at the following time points: initial presentation; during treatment at weeks 12, 18, and 30; at the end of therapy; and annually during the follow-up. All echocardiograms and related clinical assessments were reviewed. The study included 149 patients (median age 11 years; range 1-18 years). Although all patients had a reduced EF compared with their baseline echocardiogram, only 39 patients developed CTX (26\%): 43\% acute-onset, 36\% chronic early-onset, and $21 \%$ chronic late-onset. There were no statistically significant association between the frequency of myocardial dysfunction and risk factors, including age, sex, follow-up duration, cumulative DOX dose, and mediastinal XRT. Over one-third $(39 \%)$ of the patients with CTX regained normal cardiac parameters, whereas seven patients died of acute CTX. The routine uses of echocardiography to screen for ATC-induced CTX before and during CTR identified myocardial dysfunction. Early medical intervention can improve cardiac parameters. Improved screening techniques with better sensitivity and predictability are needed. ${ }^{[32]}$

\section{Financial support and sponsorship}

Nil.

\section{Conflicts of interest}

There are no conflicts of interest.

\section{References}

1. Gatta G, Capocaccia R, Coleman MP, Ries LA, Berrino F. Childhood cancer survival in Europe and the United States. Cancer 2002;95:1767-72.

2. $20^{\text {th }}$ edition of the WHO model list of essential drugs, August 2017. Available from: https:/www.who.int/medicines/publications/ essentialmedicines/en/. [Last accessed on 2019 Aug 12].

3. Von Hoff DD, Layard MW, Basa P, Davis HL Jr., Von Hoff AL, Rozencweig $\mathrm{M}$, et al. Risk factors for doxorubicin-induced congestive heart failure. Ann Intern Med 1979;91:710-7.

4. Minotti G, Menna P, Salvatorelli E, Cairo G, Gianni L. Anthracyclines: Molecular advances and pharmacologic developments in antitumor activity and cardiotoxicity. Pharmacol Rev 2004;56:185-229.

5. Erdoğan D, Yücel H, Alanoğlu EG, Uysal BA, Koçer M, Ozaydın M, et al. Can comprehensive echocardiographic evaluation provide an advantage to predict anthracycline-induced cardiomyopathy? Turk Kardiyol Dern Ars 2011;39:646-53.

6. Minotti G, Sarvazyan N. The anthracyclines: When good things go bad. Cardiovasc Toxicol 2007; 7:53-5.

7. Gianni L, Herman EH, Lipshultz SE, Minotti G, Sarvazyan N, Sawyer DB, et al. Anthracycline cardiotoxicity: From bench to bedside. J Clin Oncol 2008;26:3777-84. 
8. Salvatorelli E, Menna P, Chello M, Covino E, Minotti G. Modeling human myocardium exposure to doxorubicin defines the risk of heart failure from low-dose doxorubicin. J Pharmacol Exp Ther 2017;362:263-70.

9. Curigliano G, Cardinale D, Dent S, Criscitiello C, Aseyev O, Lenihan $\mathrm{D}$, et al. Cardiotoxicity of anticancer treatments: Epidemiology, detection, and management. CA Cancer J Clin 2016;66:309-25.

10. Tajiri K, Aonuma K, Sekine I. Cardio-oncology: A multidisciplinary approach for detection, prevention and management of cardiac dysfunction in cancer patients. Jpn J Clin Oncol 2017;47:678-82.

11. Virani SA, Dent S, Brezden-Masley C, Clarke B, Davis MK, Jassal DS, et al. Canadian cardiovascular society guidelines for evaluation and management of cardiovascular complications of cancer therapy. Can J Cardiol 2016;32:831-41.

12. Weiss RB. The anthracyclines: Will we ever find a better doxorubicin? Semin Oncol 1992;19:670-86.

13. Borchmann P, Hübel K, Schnell R, Engert A. Idarubicin: A brief overview on pharmacology and clinical use. Int J Clin Pharmacol Ther 1997;35:80-3.

14. Toffoli G, Sorio R, Aita P, Crivellari D, Corona G, Rimondi G, et al. Pharmacology of chronic oral daily administration of idarubicin. Haematologica 1997;82:1-3.

15. Anderlini P, Benjamin RS, Wong FC, Kantarjian HM, Andreeff M, Kornblau SM, et al. Idarubicin cardiotoxicity: A retrospective study in acute myeloid leukemia and myelodysplasia. J Clin Oncol 1995;13:2827-34.

16. Lothstein L, Israel M, Sweatman TW. Anthracycline drug targeting: Cytoplasmic versus nuclear - A fork in the road. Drug Resist Updat 2001;4:169-77.

17. Dabich L, Bull FE, Beltran G, Athens JW, Coltman CA Jr., Weick JK, et al. Phase II evaluation of aclarubicin in refractory adult acute leukemia: A Southwest Oncology Group Study. Cancer Treat Rep 1986;70:967-9.

18. Estorch M, Carrió I, Martínez-Duncker D, Berná L, Torres G, Alonso $\mathrm{C}$, et al. Myocyte cell damage after administration of doxorubicin or mitoxantrone in breast cancer patients assessed by indium 111 antimyosin monoclonal antibody studies. J Clin Oncol 1993;11:1264-8.

19. Thomas X, Le QH, Fiere D. Anthracycline-related toxicity requiring cardiac transplantation in long-term disease-free survivors with acute promyelocytic leukemia. Ann Hematol 2002;81:504-7.

20. Gabizon AA, Patil Y, La-Beck NM. New insights and evolving role of pegylated liposomal doxorubicin in cancer therapy. Drug Resist Updat 2016;29:90-106.

21. Gewirtz DA. A critical evaluation of the mechanisms of action proposed for the antitumor effects of the anthracycline antibiotics adriamycin and daunorubicin. Biochem Pharmacol 1999;57:727-41.

22. Menna P, Salvatorelli E, Minotti G. Anthracycline degradation in cardiomyocytes: A journey to oxidative survival. Chem Res Toxicol 2010;23:6-10.

23. Mele D, Tocchetti CG, Pagliaro P, Madonna R, Novo G, Pepe A, et al. Pathophysiology of anthracycline cardiotoxicity. J Cardiovasc Med (Hagerstown) 2016;17 Suppl 1:e3-11.

24. Lipshultz SE, Lipsitz SR, Kutok JL, Miller TL, Colan SD, Neuberg DS, et al. Impact of hemochromatosis gene mutations on cardiac status in doxorubicin-treated survivors of childhood high-risk leukemia. Cancer 2013;119:3555-62.

25. Bagnes C, Panchuk PN, Recondo G. Antineoplastic chemotherapy induced QTc prolongation. Curr Drug Saf 2010;5:93-6.
26. Caruana L, Petrie MC, Davie AP, McMurray JJ. Do patients with suspected heart failure and preserved left ventricular systolic function suffer from "diastolic heart failure" or from misdiagnosis? A prospective descriptive study. BMJ 2000;321:215-8.

27. Kupeli S. Risks and diagnosis of coronary artery disease in Hodgkin lymphoma survivors. World J Cardiol 2014;6:555-61.

28. Mulrooney DA, Nunnery SE, Armstrong GT, Ness KK, Srivastava D, Donovan FD, et al. Coronary artery disease detected by coronary computed tomography angiography in adult survivors of childhood Hodgkin lymphoma. Cancer 2014;120:3536-44.

29. Figueroa MS, Peters JI. Congestive heart failure: Diagnosis, pathophysiology, therapy, and implications for respiratory care. Respir Care 2006;51:403-12.

30. McMurray JJ, Pfeffer MA. Heart failure. Lancet 2005;365:1877-89.

31. Cardinale D, Colombo A, Bacchiani G, Tedeschi I, Meroni CA, Veglia F, et al. Early detection of anthracycline cardiotoxicity and improvement with heart failure therapy. Circulation 2015;131:1981-8

32. Moussa E, Zamzam M, Kamel A, Salah Z, Attia I, Gaber L, et al. Risk stratification and pattern of cardiotoxicity in pediatric Ewing sarcoma. J Egypt Natl Canc Inst 2017;29:53-6.

33. Grenier MA, Lipshultz SE. Epidemiology of anthracycline cardiotoxicity in children and adults. Semin Oncol 1998;25:72-85.

34. Mele D, Nardozza M, Spallarossa P, Frassoldati A, Tocchetti CG, Cadeddu $\mathrm{C}$, et al. Current views on anthracycline cardiotoxicity. Heart Fail Rev 2016;21:621-34.

35. Rathe M, Carlsen NL, Oxhøj H. Late cardiac effects of anthracycline containing therapy for childhood acute lymphoblastic leukemia. Pediatr Blood Cancer 2007;48:663-7.

36. D'Amore C, Gargiulo P, Paolillo S, Pellegrino AM, Formisano T, Mariniello A, et al. Nuclear imaging in detection and monitoring of cardiotoxicity. World J Radiol 2014;6:486-92.

37. Medical Advisory Secretariat. Magnetic resonance imaging (MRI) for the assessment of myocardial viability: An evidence-based analysis. Ont Health Technol Assess Ser 2010;10:1-45.

38. de Geus-Oei LF, Mavinkurve-Groothuis AM, Bellersen L, Gotthardt M, Oyen WJ, Kapusta L, et al. Scintigraphic techniques for early detection of cancer treatment-induced cardiotoxicity. J Nucl Med 2011;52:560-71.

39. Özdoğru I. Anthracycline-induced cardiotoxicity. Arch Turk Soc Cardiol 2014;42:274-6.

40. Iarussi D, Indolfi $\mathrm{P}$, Galderisi M, Bossone E. Cardiac toxicity after anthracycline chemotherapy in childhood. Herz 2000;25:676-88.

41. Sieswerda E, van Dalen EC, Postma A, Cheuk DK, Caron HN, Kremer LC, et al. Medical interventions for treating anthracyclineinduced symptomatic and asymptomatic cardiotoxicity during and after treatment for childhood cancer. Cochrane Database Syst Rev 2011;(9):CD008011. doi: 10.1002/14651858.CD008011.pub2.

42. Kaya MG, Ozkan M, Gunebakmaz O, Akkaya H, Kaya EG, Akpek M, et al. Protective effects of nebivolol against anthracycline-induced cardiomyopathy: A randomized control study. Int J Cardiol 2013;167:2306-10.

43. Ibrahim MA, Ashour OM, Ibrahim YF, El-Bitar HI, Gomaa W, Abdel-Rahim SR. Angiotensin-converting enzyme inhibition and angiotensin AT (1)-receptor antagonism equally improve doxorubicin-induced cardiotoxicity and nephrotoxicity. Pharmacol Res 2009;60:373-81. 
44. Bilginoğlu A, Aydın D, Ozsoy S, Aygün H. Protective effect of melatonin on adriamycin-induced cardiotoxicity in rats. Turk Kardiyol Dern Ars 2014;42:265-73.

45. Safra T, Muggia F, Jeffers S, Tsao-Wei DD, Groshen S, Lyass O, et al. Pegylated liposomal doxorubicin (doxil): Reduced clinical cardiotoxicity in patients reaching or exceeding cumulative doses of $500 \mathrm{mg} / \mathrm{m} 2$. Ann Oncol 2000;11:1029-33.

46. Lipshultz SE, Scully RE, Lipsitz SR, Sallan SE, Silverman LB, Miller TL, et al. Assessment of dexrazoxane as a cardioprotectant in doxorubicin-treated children with high-risk acute lymphoblastic leukaemia: Long-term follow-up of a prospective, randomised, multicentre trial. Lancet Oncol 2010;11:950-61.

47. Asselin BL, Devidas M, Chen L, Franco VI, Pullen J, Borowitz MJ, et al. Cardioprotection and safety of dexrazoxane in patients treated for newly diagnosed T-cell acute lymphoblastic leukemia or advanced-stage lymphoblastic non-hodgkin lymphoma: A Report of the children's oncology group randomized trial pediatric oncology group 9404. J Clin Oncol 2016;34:854-62.

48. Tien CC, Peng YC, Yang FL, Subeq YM, Lee RP. Slow infusion rate of doxorubicin induces higher pro-inflammatory cytokine production. Regul Toxicol Pharmacol 2016;81:69-76.

49. van Dalen EC, van der Pal HJ, Kremer LC. Different dosage schedules for reducing cardiotoxicity in people with cancer receiving anthracycline chemotherapy. Cochrane Database Syst Rev 2016;3:CD005008.

50. Levitt GA, Dorup I, Sorensen K, Sullivan I. Does anthracycline administration by infusion in children affect late cardiotoxicity? Br J Haematol 2004;124:463-8. 